\title{
Les gestes professionnels et le jeu des postures de l'enseignant dans la classe : un multi-agenda de préoccupations enchâssées
}

Teaching skills: a multi-agenda of embedded concerns, a set of dynamic interactions between teacher's scaffolding postures and pupils' learning ones

Dominique Bucheton et Yves Soulé

\section{OpenEdition Journals}

Édition électronique

URL : https://journals.openedition.org/educationdidactique/543

DOI : 10.4000 /educationdidactique.543

ISBN : 978-2-7535-1622-9

ISSN : 2111-4838

Éditeur

Presses universitaires de Rennes

Édition imprimée

Date de publication : 1 octobre 2009

Pagination : 29-48

ISBN : 978-2-7535-1060-9

ISSN : 1956-3485

Référence électronique

Dominique Bucheton et Yves Soulé, « Les gestes professionnels et le jeu des postures de l'enseignant dans la classe : un multi-agenda de préoccupations enchâssées ", Éducation et didactique [En ligne], 3-3 I Octobre 2009, mis en ligne le 01 octobre 2011, consulté le 22 juillet 2022. URL : http:// journals.openedition.org/educationdidactique/543 ; DOI : https://doi.org/10.4000/ educationdidactique.543 


\title{
LES GESTES PROFESSIONNELS ET LE JEU DES POSTURES DE L'ENSEIGNANT DANS LA CLASSE: UN MULTI-AGENDA DE PRÉOCCUPATIONS ENCHÂSSÉES
}

Dominique Bucheton E Yves Soulé

Lirdef, IUFM, UM2 Montpellier

\begin{abstract}
Résumé : l'article présente une synthèse de travaux de recherche du LIRDEF relatifs à l'agir enseignant dans la classe. Il développe le modèle théorique du multi-agenda mis en évidence par l'équipe pluridisciplinaire : ERT 40 de Montpellier ${ }^{1}($ 2003-2007). Il expose ensuite comment cette première modélisation a ouvert la voie à l'analyse du jeu dynamique des postures d'étayage des enseignants et des postures d'étude des élèves (travaux LIRDEF de 2007 - 2008). Elle s'achève par une étude de cas : deux débuts d'ateliers dirigés en CP.

Mots clé : gestes professionnels, postures d'étayage de l'enseignant, postures d'apprentissage, ajustement, multi-agenda
\end{abstract}

Dominique Bucheton E Yves Soulé

La nécessité d'un modèle théorique et technologique de l'agir enseignant dans la classe

\section{Un contexte idéologique et politique pressant}

Partout en Europe et en Amérique, la question de l'école et de la formation des maîtres devient une question politique et idéologique de premier plan. Les cursus de formation sont rallongés ici, raccourcis, là. Les institutions de formation sont prises dans la tourmente permanente de remises en cause, de campagnes médiatiques, de prescriptions institutionnelles, elles-mêmes soumises au maelström d'une pensée politique qui puise plus ses arguments dans des logiques économiques, technocratiques, supranationales que dans la culture accumulée par les enseignants, par les formateurs et les travaux de recherche. En même temps, l'évolution sans précédent des savoirs et des moyens de leur diffusion, la transformation dans nos pays développés des modes de vie (travail, loisirs, mentalités) questionnent en profondeur la place de l'école, ses visées et ses modes d'enseigner. Transformer la formation, accompagner les changements et les ruptures nécessaires dans l'école est une urgence pragmatique, un défi pour la recherche en éducation. Saurons-nous doter la formation d'instruments d'analyse des pratiques, performants, accessibles, cohérents, permettant d'en améliorer l'efficience? Saurons-nous ne pas perdre de vue un certain nombre de principes humanistes et démocratiques, fondateurs d'une éthique de l'instruction et de l'éducation, fondateurs aussi de la culture et de son devenir.
Les chercheurs - enseignants - formateurs qui ont contribué aux travaux présentés ici ont tous inscrit leurs recherches dans cette urgence éthique et politique visant à instrumenter mieux la formation des enseignants. Nous ne développerons cependant pas dans cet article les retombées déjà importantes pour la formation de leurs travaux.

Le modèle d'analyse de l'agir enseignant (le multiagenda) que nous présentons a en effet pour visée de fournir une grammaire complexe de concepts, permettant une analyse approfondie des situations didactiques, de leur évolution, dans leur dimension située. Il cherche à faciliter la compréhension des gestes professionnels des enseignants pour s'ajuster à la grande diversité des variables de toute situation (gérer le temps, les interactions, les savoirs, les tâches, le rapport au savoir, les attitudes des élèves, les artefacts, etc.). Il cherche à rendre compte de la manière dont diverses configurations de gestes (des postures) peuvent générer différentes dynamiques cognitives et relationnelles dans la classe.

\section{La transposition nécessaire d'un ensemble très riche de travaux théoriques}

L'analyse du travail de l'enseignant n'est pas nouvelle. Depuis plus de cinquante ans, elle a fait l'objet de nombreux travaux très divers et très féconds qui ont exploré une multiplicité d'aspects. Cependant, l'ensemble très vaste de ces travaux a été peu diffusé, peu vulgarisé et surtout peu transposé et 
mis en perspective pour la formation des enseignants. Le modèle du multi-agenda s'est donc élaboré dans une culture et un contexte scientifique d'autant plus riche que les chercheurs de l'équipe relevaient de disciplines différentes (psychologie sociale, sciences $\mathrm{du}$ langage, didactique de plusieurs disciplines, didactique générale, sciences de l'éducation). Nous ne pouvons présenter ici le large arrière-plan théorique dans lequel le modèle a largement puisé ${ }^{2}$.

Force est de constater que peu nombreux sont les formateurs ou les enseignants capables aujourd'hui de synthétiser l'ensemble de ces travaux pour conduire une analyse de pratiques à la hauteur de ce que l'on sait des différentes facettes de l'agir enseignant. Le résultat en est que les étudiants, les stagiaires reçoivent en formation des conseils, des prescriptions, des éléments de théorisation, très éclatés, voire contradictoires, dont la complémentarité et la cohérence sont loin d'être évidentes, ce qui contribue à augmenter parfois leur désarroi devant la difficulté croissante du métier.

Une première visée pragmatique de nos travaux a donc été de contribuer à la transposition, la didactisation et la mise en perspective pour la formation, de l'ensemble éclectique de ces divers travaux de recherche en sciences humaines afin de résoudre un certain nombre de problèmes qu'en tant que formateurs et chercheurs nous avions identifiés.

\section{Des obstacles théoriques et praxéologiques bien identifiés}

La commande d'une recherche à caractère technologique est en effet d'identifier des problèmes, de les analyser et d'apporter les instruments théoriques et technologiques pour les résoudre. Nous en avons repéré cinq, très urgents pour la formation et son évolution.

Rompre en formation et dans la recherche avec la partition didactique / pédagogie

Dans l'ordinaire de l'agir dans la classe, les contenus d'enseignement (que ce soit des objets de savoir, des méthodes, des pratiques, des attitudes, etc.) sont indissociables et même inextricablement liés aux conditions de leur enseignement et appropriation. Dans les curricula et dispositifs de forma- tion, ils sont cependant dissociés, comme ils le sont d'ailleurs dans les diverses listes de compétences professionnelles. Or, se préparer à agir dans la classe, c'est apprendre à combiner les différentes variables d'une situation d'enseignement et d'éducation. Dans les instituts de formation de maîtres en France, les didacticiens des différentes disciplines rencontrent peu leurs collègues spécialistes de "pédagogie ", diversement appelée formation générale ou transversale. Au stagiaire dans sa classe de se débrouiller pour tisser les liens entre les différents points de vue. Ainsi des questions telles que l'autorité, la violence, la gestion du temps ou l'usage du tableau peuvent être traitées sans que soient évoquées les situations d'enseignement, les rapports au savoir ou à l'institution qu'elles mettent souvent en scène (ou en pièces!).

Lélève et le maître: des personnes et pas seulement des sujets épistémiques

S'il est contre-productif de séparer didactique et pédagogie, de séparer l'appropriation des savoirs de leurs contextes d'apprentissage, considérer les élèves et les maîtres simplement comme des sujets épistémiques, des sujets déréalisés, ne facilite pas la compréhension de la diversité des obstacles que posent les apprentissages. Nous postulons au contraire que pour comprendre le développement cognitif de sujets en formation, il est nécessite de ne pas dissocier ce développement du développement langagier, psychosocio-affectif et identitaire (Bucheton 1994). Élèves et maîtres sont à considérer pour la compréhension de leurs relations et comportements, comme des personnes, porteuses d'une histoire, d'une culture, d'un rapport à l'institution, d'un rapport au savoir enseigné. Elles vivent en classe des émotions et manipulent des registres langagiers, identitaires et cognitifs multiples. La vie est aussi dans l'école. C'est elle qui donne tout son sens au désir d'apprendre et de grandir.

Identifier la singularité de l'agir de l'enseignant, sa créativité au coeur de formes sociales et scolaires pérennes

La singularité et la créativité de l'action du maître sont très vite identifiées par les élèves (Durand 1996). En revanche elles sont encore mal expliquées théoriquement. Tel est le troisième challenge auquel 
nous avons travaillé en prenant notamment appui sur les travaux ergonomiques, en particulier la clinique de l'activité d'obédience française (Clot 1999). Après Bakhtine (1984), ces travaux montrent comment l'agir et la parole humaine s'inscrivent dans une culture, des cadres d'expérience partagés (Goffman 1991) et en même temps les dépasse pour les recréer et les développer. Pour l'école, cet agir est stabilisé dans des formes scolaires descriptibles, historiquement et socialement construites (Thévenaz-Christen 2005). La question est alors de comprendre mieux où s'origine la dimension irréductiblement singulière de cet agir, ce que parfois on appelle le charisme propre de l'enseignant. Quels en sont les ressorts principaux : les logiques d'arrière-plan (Bucheton 2009 a p. 27-68) plus ou moins conscientes?

Face à cette question, décisive pour la formation, plusieurs travaux de recherche de l'équipe ont pu apporter des éléments de réponse. Ils ont mis en évidence divers éléments constitutifs de ces logiques d'arrière-plan : le rapport au savoir enseigné (Broussal 2006), la représentation plus ou moins déficitaire des possibles intellectuels des élèves (Brunet § Liria 2004). Ces logiques orientent, colorent à l'identique les décisions prises dans l'urgence des ajustements provoqués par la dynamique de l'action et ses imprévus (Jean 2008).

\section{Donner un statut aux divers langages dans la classe}

Une quatrième perspective, et non la moindre du travail présenté, est celle qui concerne la place du langage dans l'agir de la classe. Dans le numéro 30 de la revue Repères / INRP consacré au langage dans la formation (Bucheton, Bronner, Broussal, Jorro, Larguier 2004), nous attirions l'attention sur la dimension trop minorée, voire impensée du rôle du langage dans les cursus de formation des maîtres. Le langage, pourtant, façonne l'arrière-plan épistémologique de la discipline enseignée, ses modes d'énonciation, spécifiques (Jaubert, Rebière 2001). Il est le vecteur principal du travail partagé et des relations entre le maître et les élèves. Il est un révélateur, une fenêtre sur l'avancée des significations en train de s'élaborer (Bucheton 2007), le levier principal du développement de la réflexivité et de la conceptualisation visée (Chabanne, Bucheton 2002). Nous insistions aussi dans cet article sur la diversité sémio- logique des artefacts et langages à l'œuvre dans la classe, sur l'importance de ce que Jorro (2002-2004) appelle le corps parlant de l'enseignant.

Rendre compte de l'évolution des significations partagées pendant la leçon, théoriser les imprévus

Comprendre comment le sens avance cahin-caha dans la classe, au travers de toutes sortes d'imprévus est un rude défi! De nombreux didacticiens se sont attaqués à ce problème central ${ }^{3}$. Les concepts de topogénèse, mésogénèse, chronogénèse élaborés par Sensévy \& Mercier, 2007, la notion de microgénèse située (Saada-Robertet § Baslev 2004) ont ouvert la voie. Nous abordons cette question par l'étude du rapport entre les gestes des enseignants et les gestes d'étude des élèves. Nous postulons en effet que l'émergence et le développement des significations (des références) plus ou moins partagées par l'ensemble de la classe et du maître sont tributaires de l'engagement réciproque des acteurs. On ne peut donc dissocier l'étude du développement des significations pendant le cours, de celle des postures et gestes qui les actualisent et les permettent. Derrière ces gestes et postures, une double dynamique se joue. Elle manifeste côté maître et côté élèves des formes, des degrés d'engagement dans les tâches, des finalités parfois fort différentes: par exemple faire le travail demandé pour comprendre un problème, pour se débarrasser de la tâche ou pour faire plaisir au professeur, n'a pas le même sens et ne produit pas le même apprentissage chez l'élève. Cette dynamique complexe est-elle descriptible? Est-elle modélisable? Les premiers résultats (Soulé, Bucheton 2007, Dupuy 2009) montrent des régularités identifiables dans les corrélations et configurations de gestes et postures entre le maître et les élèves. Nous approfondirons ce dernier point plus loin.

Cette dynamique singulière de l'avancée d'une leçon est émaillée d'imprévus ${ }^{4}$ qui surgissent constamment dans le cours de l'action. Ils sont des indicateurs de ce cheminement non tranquille de la construction des significations au travers d'engagements instables de la part des acteurs dans l'action. (Jean 2008).

Tout formateur en a fait souvent l'expérience lors de visites : préparer la classe selon une chronologie 
d'objectifs, de tâches pour y parvenir, de dispositifs et artefacts pour en préparer la mise œuvre judicieuse ne règle pas le problème de la tension entre le déroulement programmé et le surgissement nécessaire de ces imprévus. Enseigner c'est apprendre à gérer et tirer profit de la zone d'incertitude inhérente au partage d'une activité entre des humains très différents par l'âge et la culture. C'est se préparer à accueillir et à traiter comme événements porteurs de sens ce que les élèves apportent, ce qu'ils savent déjà ou ont compris autrement dans leur expérience passée. Mais ces ajustements ne doivent pas faire perdre de vue la nécessité de maintenir l'orientation générale de la trame du récit préparé, pour y incorporer les significations temporaires afin d'aller jusqu'à la chute de la leçon. Elle assoit le sens en reprenant, réinterrogeant ses étapes intermédiaires. La métaphore de la classe comme un texte qui déroule un récit à plusieurs voix (Decron 2007), empruntée à Labov (1976), mais aussi à Bruner (2000) permet de penser l'événement c'est-à-dire l'imprévu comme une des sources même de la dynamique du sens se construisant. Une dynamique qui naît de l'inter-élaboration, évolution des significations entre les acteurs et les objets manipulés. La représentation de l'imprévu comme révélateur d'une préparation insuffisante ou d'une professionnalité chancelante se doit d'être mise en doute ou tout au moins interrogée.

Conclusion intermédiaire : la nécessité d'un modèle fédérateur, heuristique, qui soit praxéologique mais non normatif

La mise en perspective d'un large empan de cadres théoriques pour analyser des situations de classe et les problèmes didactiques et pédagogiques qu'elles posent ont permis l'élaboration d'une modélisation de l'agir enseignant dans la classe. La nécessité de la vulgarisation de ce modèle auprès de publics d'enseignants et formateurs nous a conduit à accorder la plus grande attention au choix des termes désignant les préoccupations des enseignants. Nous avons sélectionné un réseau réduit de concepts au fort pouvoir heuristique pouvant permettre à des chercheurs, des formateurs, des stagiaires et enseignants de penser et travailler ensemble sur des problèmes identifiés. Nous avons cherché à élaborer un modèle qui soit :

- un modèle fédérateur : il trouve sa source dans une culture théorique vaste dont il théorise les rencontres et les filiations : travaux en didactiques des disciplines, en psychologie cognitive et sociale, en ergonomie, apports de la phénoménologie, de la sociologie de l'école, ancrage fort dans l'interactionnisme, l'ethnométhodologie, les sciences du langage dans leur articulation aux contextes de l'école, mais aussi ancrage dans les théories de l'action située,

- un modèle heuristique: son but est de permettre de dérouler la pelote de ce qui se joue dans la classe pour pouvoir mieux rendre compte de la complexité et l'épaisseur de l'agir du maître,

- un modèle praxéologique et non normatif : l'histoire de ce modèle est inscrite dans les préoccupations de la formation des enseignants. Sa vocation est de l'instrumenter mieux en construisant un réseau des concepts, un langage commun, non pour formater des pratiques mais pour les questionner en profondeur. Son écriture se veut donc simple, visuelle, accessible. Les concepts de base - gestes professionnels, ajustement, pilotage, tissage, étayage, atmosphère, postures - ont été nommés à partir de termes à fort pouvoir métaphorique. La métaphore est un instrument poétique puissant pour donner à comprendre, à imaginer, à relier des univers d'expérience, d'émotion et de réflexion, disjoints.

\section{Le modèle du multi-agenda, une architecture de cinq macro-préoccupations conjuguées (travaux de 2004-2007 de l'ERTE 40)}

Cinq préoccupations centrales constituent selon nous la matrice de l'activité de l'enseignant dans la classe, ses organisateurs pragmatiques dominants (Pastré, Mayen § Vergnaud 2006) : 1) piloter et organiser l'avancée de la leçon, 2) maintenir un espace de travail et de collaboration langagière et cognitive, 3 ) tisser le sens de ce qui se passe, 4) étayer le travail en cours, 5) tout cela avec pour cible un apprentissage, de quelque nature qu'il soit. Ces cinq préoccupations qui se retrouvent de la maternelle à l'université, sont cinq invariants de l'activité et constituent le substrat des gestes professionnels. Par geste professionnel, nous désignons de manière métaphorique l'action de l'enseignant, l'actualisation de ses préoccupations. Le choix du terme geste traduit l'idée que l'action du maître est toujours adressée et inscrite dans des codes. Un geste est une action de communication 
inscrite dans une culture partagée, même a minima. Il prend son sens dans et par le contexte scolaire.

Nous postulons que ces cinq préoccupations sont les piliers autour desquels s'élaborent l'agir ordinaire dans la classe, les savoirs professionnels, l'expérience et les compétences.

Ces cinq préoccupations, présentées dans la figure 1 page suivante ne sont jamais isolées.

Elles sont systémiques dans le sens où elles coagissent, rétroagissent les unes avec les autres : décider ou non de rectifier la réponse d'un élève s'inscrit aussi bien dans ce qu'on appellera étayage, que dans ce qui relève de l'atmosphère et de la gestion des faces, comme dans le souci de ne pas trop retarder le pilotage de la leçon.

Elles sont modulaires : en début de cours le souci atmosphérique de construire un climat de classe pour engager les élèves dans l'action est fortement lié à une nécessité de pilotage : cadrer la séance et ses enjeux. L'étayage par contre peut y être plus ténu.

Elles sont hiérarchiques. Il est clair que selon les dispositifs ou enjeux de la situation mise en place (une sortie scolaire en début d'année par exemple) le souci de mettre en travail des savoirs spécifiques peut passer au second plan parce que l'objectif central est avant tout de créer les conditions relationnelles de la poursuite du travail.

Elles sont dynamiques. Leur mise en synergie, leur organisation interne évoluent pendant l'avancée de la leçon. Elles sont plus ou moins embryonnaires, développées et précises selon le degré d'expérience et selon la culture des enseignants. Le jeu disponible des gestes professionnels pour installer ou maintenir une atmosphère ou simplement son autorité, est ainsi très variable d'un maître à l'autre. On verra plus loin comment le principe de modularité (emprunté à la psychologie cognitive) explique l'organisation de configurations de gestes en postures.

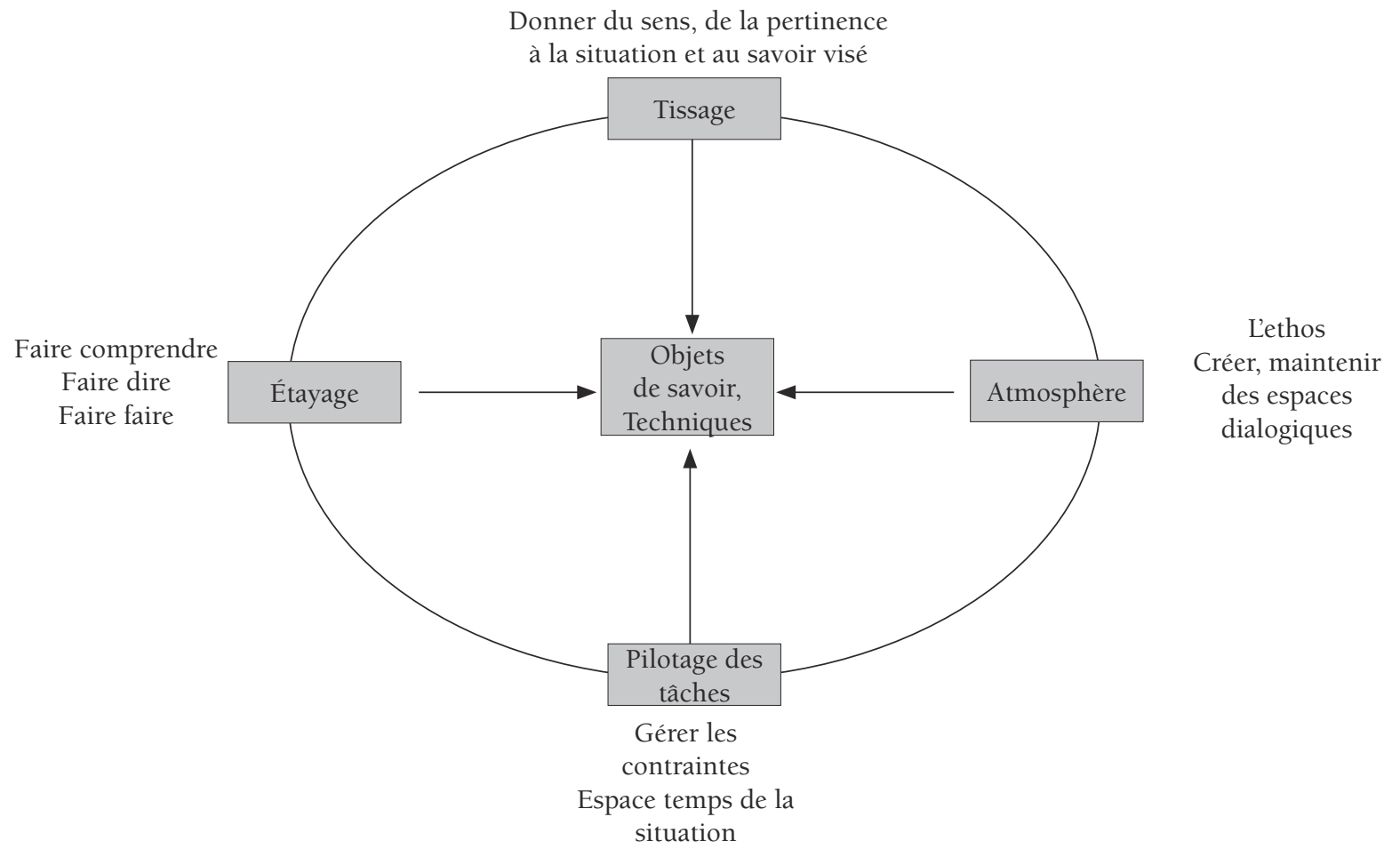

Figure 1: un multi-agenda de préoccupations enchâssées 


\section{Le pilotage de la leçon :}

Sa visée centrale est d'organiser la cohérence et la cohésion de la séance, d'assurer la chronogénèse de la leçon, de passer d'un début où on se contente de cadrer la visée de savoir parfois de problématiser, parfois d'organiser la dévolution du savoir à travailler jusqu'à une clôture où l'objet de savoir sera plus ou moins conceptualisé, nommé, institutionnalisé quelquefois par une trace écrite. Ce parcours nécessite d'organiser les tâches, les instruments de travail, la disposition spatiale, les déplacements autorisés ou non. Cette préoccupation, centrale pour les débutants, s'inscrit dans un ensemble de contraintes plus ou moins négociables (la classe, les habitus de l'établissement, les prescriptions et programmations). La temporalité factuelle de la leçon inscrite sur la montre et l'emploi du temps est en fait la résultante de nombre d'autres temporalités institutionnelles, didactiques, subjectives qui font en partie l'objet de la préparation de la leçon et qui sont l'objet de dilemmes constants pendant son déroulement. Aurai-je le temps de faire cet exercice? Combien de temps leur faudra-t-il pour écrire cette phrase? Dois-je avancer même s'ils n'ont pas terminé? Allons-y pour une digression, une anecdote, elle me fera gagner du temps en explication. Stop! je m'arrête : ils ne me comprennent pas, ils sont ailleurs. Stop, là il ne faut pas que je rate cette remarque. Cette gestion du temps, du rythme (dilatation, accélération, pauses) s'accompagne d'une autre préoccupation non moins forte qui est pour le maître celle de ses déplacements et de ceux des élèves, de ses gestes corporels, de la maîtrise des artefacts de base : manuels, tableaux, affichages, disposition des tables et place des élèves. La maîtrise progressive du pilotage général dès lors que celui-ci n'est plus une angoisse constante, ouvre à l'enseignant la possibilité de se rendre disponible pour des moments de communication particulièrement heureux où le temps de la classe semble suspendu : des micro-scénarios improvisés, des moments d'attention conjointe provoqués par un événement qui vient de surgir et dont on se saisit (l'art du « kairos » dont parle Jorro 2004). Le pilotage conjugue ainsi la dynamique tranquille de la durée, nécessaire à l'incorporation des savoirs avec la force de l'instant, de la rencontre qui déstabilise et avive la curiosité.

\section{L'atmosphère}

L'atmosphère c'est l'espace intersubjectif qui organise la rencontre intellectuelle, relationnelle, affective, sociale entre des individus confrontés à une situation contenant des enjeux à gérer en commun. Cet ethos (Maingueneau 1997) est le liant dans lequel baignent les interactions et qui en même temps les colore d'une certaine tonalité : sérieuse, ludique, tendue, ennuyeuse, voire inquiétante etc.

Les maîtres mots en sont relations, engagement, éthique, langages, espace pour penser.

Récemment, deux écrivains, Frank Mac Court dans Teacher man, (2006), et Daniel Pennac dans "Chagrins d'école », (2007), racontant l'un et l'autre leurs souvenirs d'enseignants dans des contextes difficiles, les lycées professionnels du Bronx pour le premier, des élèves en rupture pour Pennac, mettent tous les deux en scène cette atmosphère décisive pour capter l'attention des élèves, les faire entrer dans l'indicatif présent de la classe (Pennac), susciter et maintenir leur engagement affectif, relationnel, intellectuel. Ils y décrivent ces joies et chagrins partagés entre les maîtres et les élèves, cet art difficile de la gestion des faces, de la négociation des rôles, des rituels pour contractualiser les places, les statuts de chacun. Ils racontent les ratés, les marges de manœuvre encore plus réduites quand on est un enseignant contractuel. Ce concept d'atmosphère concentre ainsi un certain nombre de travaux relevant de l'interactionnisme symbolique, de l'ethnométhodologie, de l'analyse des discours s'intéressant aux interactions langagières, intersubjectives, en classe (François 1990). Il fait aussi la part belle au rôle de l'émotion vecteur de la rencontre, de l'engagement, du désir, mais aussi de la peur et de l'inhibition (Damasio 1995), (Berthoz 2003). Tant il est vrai qu'il ne peut y avoir d'apprentissage sans désir, sans appétit, ni motif!

Ce concept prend également en compte les travaux de la psychologie sociale (Perret Clermont 1988) qui ont montré l'importance du conflit sociocognitif pour dépasser, déplacer l'espace de sa propre pensée.

Mais le concept renvoie aussi aux travaux de l'équipe de Blanchard Laville (2003) qui ont su montrer 
par exemple comment la dimension psychanalytique peut expliquer des assignations de place, spécifiques (fils chéri, bouc émissaire pour certains élèves).

Les gestes d'enrôlement, de maintien de l'attention, (Bruner 1984), le souci de la gestion de la face de l'élève lors de l'évaluation, l'écoute attentive, les plaisanteries ou réprimandes en sont l'ordinaire.

Ajoutons aussi combien ces gestes d'atmosphère relèvent d'une éthique professionnelle : laisser aux élèves un espace de parole pour parler, penser, apprendre et se construire, leur apprendre à être à l'écoute de l'autre. Ainsi lire un texte d'élève sans prendre le stylo rouge mais d'abord simplement pour entendre ce que l'élève, l'enfant vient de dire, relève de cette atmosphère et de cette éthique qui fait de l'élève une personne pour laquelle l'enseignant a du respect mais aussi un devoir et un désir : celui de le faire progresser.

La gestion de l'atmosphère de la classe se joue souvent conjointement ou successivement sur plusieurs scènes: scène collective et frontale, scène d'arrière-plan où se règlent les petits accords ou conflits entre les élèves, scène duale entre le professeur et un élève, scènes de groupes ou les élèves sont en autonomie collective, scène d'atelier où le maître s'est rendu disponible pour quatre ou six élèves, scène intérieure que l'enseignant surveille en observant le regard, les jeux de mains ou les tics de ses élèves. Chacune de ces scènes organisant une atmosphère cognitive et langagière différente et permettant des formes d'engagement, une présence, différentes pour les élèves et le maître.

Cette atmosphère est sous la responsabilité de l'enseignant. Il est clair que les élèves tentent parfois de faire se détériorer ce climat de classe et d'en reprendre le leadership. Les effets cognitifs de ces « intempéries » de la classe sont probablement de première importance. Les travaux de la psychologie cognitive attirent notre attention sur le fait que la pensée se développe dans un certain climat intérieur du cerveau. Jean Didier Vincent (2007) parlant de l'activité cérébrale parle d'un État Central Fluctuant : un état parce que toujours en devenir, en équilibre instable, central, parce que son activité renvoie à la totalité de notre système nerveux qui déploie notre présence au monde et fluctuant comme hautement labile et sensible aux moindres intempéries. On peut imaginer qu'atmosphère de classe et climats intérieurs des cerveaux des élèves sont fortement corrélés.

\section{Le tissage}

Par tissage nous nous référons à l'activité du maître ou des élèves pour mettre en relation le dehors et le dedans de la classe, la tâche en cours avec celle qui précède ou qui suit, le début avec la fin de la leçon. Ces opérations affichent une constance de $7 \%$ des gestes des enseignants dans de nombreuses situations analysées en primaire; elles montent à plus de $40 \%$ dans l'enseignement technique.

Les travaux sur le CP (Brunet Liria, Bucheton 2004-2005) montrent que les bons élèves tissent eux-mêmes les liens laissés à l'état implicite par l'enseignant. Ils savent nommer les tâches et objets de savoirs qu'elles travaillent et sont capables d'en comprendre le pourquoi et la succession. Les élèves moyens peuvent souvent retrouver les tâches en termes de "faire », en repérer l'ordre; les élèves faibles, quant à eux n'arrivent ni à les nommer ni à en retrouver l'ordre bien qu'ils les aient accomplies de manière routinière un très grand nombre de fois.

Ainsi ce concept de tissage se veut une métaphore de l'activité centrale du cerveau qui consiste à multiplier dans une alchimie encore bien obscure, les synapses entre les neurones, pour favoriser le renforcement des traces, câblages, associations, sensibilisations. Il renvoie surtout aux travaux de la sociologie et l'anthropologie culturelle qui montrent comment tout savoir, toute expérience nouvelle, tout discours (Bakhtine 1984) s'inscrit dans une continuité, dans des genres, des habitus déjà construits, des stéréotypes, des formes de l'imaginaire déposées dans la culture et l'histoire. Tisser c'est d'abord raviver les empreintes que l'expérience a laissées. L'école très souvent sous-estime cette dimension ou la gère mal. Les savoirs y sont souvent trop vite décontextualisés, déréalisés ou cloisonnés. Ce qui ne pose pas problème aux bons élèves qui refont en partie euxmêmes ou avec l'aide des parents les contextualisations, décontextualisations et recontextualisations nécessaires. Tisser c'est réveiller, raviver des traces déjà là, (les fameux brain-storming) pour planter le décor, construire le milieu d'une séance. Ainsi, 
les moments d'ouverture de séance, où la mémoire didactique (Mathéron 2000) est ravivée comme ceux de clôture sont des moments clés du tissage.

\section{L'étayage : un organisateur central de l'agir enseignant}

L'étayage est, dans le modèle proposé, un concept central, hiérarchiquement supérieur aux autres. On verra plus loin qu'il peut être pensé comme l'organisateur principal de la co-activité maître-élèves.

Nous avons repris à Bruner, ce concept d'étayage pour désigner toutes les formes d'aide que le maître s'efforce d'apporter aux élèves pour les aider à faire, à penser, à comprendre, à apprendre et à se développer sur tous les plans. La métaphore de Bruner « scafolding » montre bien toute l'ambiguité de cette relation d'aide. Indispensable, mais aussi vouée à disparaître.

«Scafolding », c'est l'échafaudage qu'on enlève quand la maison est construite, mais c'est aussi l'étai pour creuser des galeries dans la mine. Il a besoin d'être fiable, durable, il nécessite la confiance.

Bruner parlant d'apprentissage manuel avec de jeunes enfants insiste particulièrement sur le maintien de l'orientation de l'activité, sur le pointage de la difficulté ou de l'erreur, sur la nécessité parfois de gestes de monstration. Après Vygotsky, il insiste beaucoup sur la dissymétrie fondamentale de la place qu'occupe celui qui sait, qui enseigne ou accompagne. Celui dont le rôle est d'être un passeur, cherchant des gués, tenant la main de l'apprenant pour les franchir, mais aussi celui qui doit souvent contrôler sa frustration et se retenir d'expliquer, ou de faire à la place de l'élève (le geste d'impatience du maître qui pose la question et n'attend pas la réponse).

Les gestes d'étayage offrent une grande diversité de réalisation selon les disciplines, l'avancée de la leçon, l'hétérogénéité plus ou moins grande du public, la nature de la tâche ou des savoirs que celle-ci cherche à mettre en évidence. Ils actualisent toutes sortes de dilemmes. Ils traduisent des décisions dont l'équilibre est fragile. Leur examen attentif et comparé, dans différentes situations d'enseignement, montre qu'ils sont le lieu même où s'articule ce qu'on pourrait appeler le didactique et le pédagogique. Ils sont toujours didac- tiques au sens où ils visent un but didactique spécifique, ils sont pédagogiques au sens où ils sont l'instrument pour y parvenir. L'étayage est donc au cour du métier, impulse, régule la dynamique de ce qui se joue dans la classe. Mais une dynamique dont les règles demeurent encore très obscures. L'enseignant gagne quand l'élève gagne dit Sensévy (2008). La compréhension des règles cachées de ce jeu partagé, est l'objet de nombreux travaux actuels. Elle est au centre des préoccupations du réseau international de recherche : OPEN (contrat 2008-2012). La deuxième matrice conceptuelle que nous présenterons cherche à éclaircir quelques-unes de ces dynamiques.

\section{Les savoirs visés : une cible floue}

La cible visée par la situation d'enseignement/ apprentissage est assez souvent floue, peut-être l'estelle nécessairement. La problématique des objets de savoir, des attitudes, du savoir-être, savoir-faire a donné lieu à une littérature considérable en sciences humaines. Enseigne-t-on des concepts, des techniques, des attitudes, des méthodes, des stratégies, des comportements scolaires, sociaux, des pratiques sociales, des formes d'adaptation et d'ajustement aux situations etc. ? Chevallard (1999), dans son quadruplet distinguant les tâches, des techniques pour les faire, des technologies : les discours et conceptualisations qui sous tendent l'action et les théories dont ces technologies sont issues ou qu'elles mettent en évidence, tente de mettre un peu d'ordre. Mais ce modèle anthropologique ne prend pas assez en compte la dimension socio-affective et expérientielle, constitutive du rapport au savoir et de son évolution.

Si on ajoute à cela le fait que dans le primaire les maîtres enseignent des savoirs pour lesquels ils ont une culture très réduite et parfois une expérience douloureuse, on comprend alors pourquoi le savoir enseigné est souvent pensé par les élèves et les maîtres plus comme un "faire quelque chose » (Crinon, Marin, Bautier 2008) que comme la réponse à une question qu'on s'est posée. Les jeunes stagiaires « font » le programme sans être en mesure de s'autoriser à en questionner la pertinence (Bucheton Chabanne 2002).

Les savoirs visés sont aussi fondamentalement hétérogènes, tout particulièrement en didactique 
du français. Leur objectivation, leur conceptualisation intermédiaire ou institutionnelle en est rendue d'autant plus difficile.

Donnons un exemple pris dans une séance de lecture-écriture en maternelle. L'enseignante veut faire écrire « alligator», mot vu dans un album. On constate que toutes sortes de savoirs sont convoquées, parfois nommés.

1) des concepts tels que « son », « syllabe », « mot », sont nommés plusieurs fois,

2) des techniques (des gestes d'étude) sont mises en place pour les repérer, discriminer auditivement le $[\mathrm{G}] \mathrm{du}[\mathrm{K}]$, les associer (on frappe dans ses mains), on les cherche dans les «mots » affichée sur le mur,

3) des savoirs culturels: " alligator, je sais le lire parce que c'est dans le titre de la couverture de l'album, dit un élève ».

4) une pratique sociale (savoir écrire) est verbalisée. Elle constitue l'arrière-plan socioaffectif et identitaire de la leçon : "regarde, dit une élève en montrant à la caméra sa feuille, je sais déjà écrire tout ça! »

Mais pour entrer dans la compréhension et la manipulation de l'écrit, c'est l'ensemble de ces savoirs que l'élève doit s'approprier en les conjuguant.

La question de l'identification des savoirs visés, de leur complexité, de leur nécessaire imbrication, de leur évolution en cours d'action, en même temps que celle de la possibilité pour les élèves d'y avoir accès (la Zone de Proche Développement de Vygotsky1985) est une question centrale pour déterminer la nature de l'étayage apporté par l'enseignant.

\section{Postures d'étayage des maîtres, postures d'apprentissage des élèves : un jeu dynamique (travaux de 2007-2008)}

Nous présentons maintenant les nouvelles recherches conduites à partir de la matrice conceptuelle du multi-agenda. Elles visent à identifier des régularités dans les co-ajustements de l'agir du maître avec celui des élèves. Ces travaux, réalisés à l'occasion d'un programme de recherche LIRDEF -
INRP sur l'écriture au CP, co-piloté par D. Bucheton et Yves Soulé (contrat 2007-2008) se poursuivent actuellement dans le cadre d'une recherche sur la mise en ouvre d'ateliers dans les classes des Réseaux Ambition Réussite (ex ZEP) de collège: (programme 2008-2009, soutenu par le centre A. Savary de l'INRP).

Les premiers résultats de ces divers travaux laissent apercevoir des motifs réguliers dans les ajustements des gestes professionnels des maîtres. Ils contribuent à la compréhension des heurts, bonheurs, malheurs de la communication (François 1990) et de la co-construction de significations pendant la classe. Ils ont permis l'élaboration d'une deuxième matrice conceptuelle: celle du « jeu des postures d'étayage » du maître.

Nous présentons d'abord la méthodologie adoptée puis le jeu des postures observées chez les maîtres. Dans un second temps, au travers de l'étude comparative de deux débuts d'ateliers d'écriture en CP nous analysons les premières configurations observées entre les postures d'étayage de deux maîtres et les postures et gestes d'étude des élèves. Ce dernier exemple a pour but de donner un aperçu des méthodes d'analyse, du type de résultats attendus, comme de leurs limites.

\section{Éléments de méthodologie : le principe de la comparaison}

La méthodologie d'analyse adoptée s'appuie sur le principe de la comparaison. Après avoir identifié les éléments communs du « genre scolaire » observé, nous cherchons à repérer les zones de variation, d'ajustement dans l'action pour tenter d'en comprendre les raisons et ceci de deux façons : 1) en observant l'activité des élèves en regard de cet agir, 2) en confrontant ce que nous avons constaté avec ce qu'en disent les enseignants concernés lors d'entretiens d'auto-confrontation à la vidéo de la séance. Le dispositif étudié : l'atelier dirigé, sorte de mini-classe, permet une observation quasi exhaustive des interactions des acteurs.

Nous avons dans un premier temps enregistré et analysé plusieurs séances d'ateliers d'écriture en $\mathrm{CP}$ en début d'apprentissage du lire-écrire entre octobre et décembre. Nous avons ensuite choisi de travailler d'un 
point de vue clinique et qualitatif, linguistique, didactique et ergonomique sur deux enseignantes également anciennes dans le métier, exerçant chacune dans des classes très hétérogènes. La durée de l'atelier n'excède pas une demi-heure. Le regroupement des six élèves dans chacun des ateliers s'est fait sur la base de cette hétérogénéité. Aucune consigne spécifique, exceptée celle de ne pas trop dépasser la demi-heure, n'a été donnée aux enseignantes. Les données ont été collectées dans des classes ordinaires à l'intersession d'un stage de formation continue de deux fois deux jours. Les séquences filmées ont donné lieu à une analyse collective lors des deux dernières journées de stage un mois plus tard. Des auto-confrontations immédiates et des entretiens ont accompagné la prise de vue ${ }^{5}$.

L'analyse s'est opérée en plusieurs rangs :

1) Les synopsis des séances : la succession des scénarios et leur temporalité précise.

2) L'identification pour chacun de ces scénarios des gestes professionnels et savoirs mobilisés à partir du cadre conceptuel du multi-agenda,

3) L'identification de configurations de gestes (des postures) tant chez les élèves que chez le maître, à partir d'analyses linguistiques sur les formes de l'interpellation et leurs effets.

4) la mise en perspective des analyses avec ce que les enseignants disent de la séance dans les auto-confrontations.

5) La reprise ultérieure de ces résultats en nous intéressant de plus près aux gestes évaluatifs et aux formes énonciatives de l'enseignant (Soulé, Bucheton 2007).

6) La confrontation à plusieurs reprises des résultats obtenus à d'autres situations d'ateliers chez des enseignants débutants (stagiaires IUFM) pour vérifier la pertinence de la grille d'analyse des postures d'étayage construite à partir des pratiques des enseignants experts.

\section{Premiers résultats : des gestes de métier installent vite un genre commun et son pilotage :}

L'atelier dirigé d'écriture pendant lequel le maître reste avec 5 ou 6 élèves qui écrivent individuellement leur texte pendant une demi-heure alors que les autres sont en autonomie, est très peu familier pour les enseignants ou les élèves de CP. Pourtant, dès les premières mises en œuvre, on retrouve d'emblée un synopsis commun comportant 3 ou 4 scénarios : A) la négociation du texte (en général une phrase), B) un long temps sur les deux ou trois premiers mots, C) l'écriture de la fin de la phrase, D) la clôture de l'atelier. Autrement dit les lois de l'atelier dirigé comme «genre» s'imposent vite. Nous interprétons cette organisation dynamique commune (pilotage général) comme un indicateur chez les enseignants experts de la maîtrise de gestes de métiers montrant des préconstruits professionnels communs pour gérer la tache et surtout le temps, notamment les ralentissements en B ou accélérations en C qu'elle demande. Cependant le pilotage de ces ateliers révèle aussi des variations signifiantes relatives à la durée des scénarios de début et de clôture très courts chez l'une des deux enseignantes observées, beaucoup plus longs chez l'autre. Mais avant de poursuivre la présentation de ces résultats, il nous faut revenir sur des travaux plus anciens.

\section{Retour sur le terme posture aujourd'hui très à la mode :}

Le concept de posture tel que nous l'utilisons depuis fort longtemps pour des travaux relevant de la didactique du français, (Bucheton, Bautier 1997) prend sa source dans la théorie des concepts en actes de Vergnaud (1996).

La définition que nous avons donnée du terme "posture » est la suivante : "Une posture est un schème préconstruit du "penser-dire-faire ", que le sujet convoque en réponse à une situation ou à une tâche scolaire donnée. La posture est relative à la tâche mais construite dans l'histoire sociale, personnelle et scolaire $d u$ sujet. Les sujets disposent d'une ou plusieurs postures pour négocier la tâche. Ils peuvent changer de posture au cours de la tâche selon le sens nouveau qu'ils lui attribuent. La posture est donc à la fois du côté du sujet dans un contexte donné, mais aussi de l'objet et de la situation, ce qui en rend la saisie difficile et interdit tout étiquetage des sujets (Bucheton 1999 - Bucheton 2006). Les travaux que nous avons menés sur les postures des élèves dans des conduites de lecture littéraire ou d'écriture montrent cependant que les élèves 
les plus en réussite sont ceux qui disposent d'une gamme plus variée de postures. Ils savent changer de posture dans la difficulté. On constate aussi qu'à niveau scolaire égal, devant la même tâche, la variété plus ouverte des postures est corrélée statistiquement à l'appartenance à un milieu favorisé. On peut alors faire l'hypothèse que ce n'est pas l'école qui construit la diversité et l'ouverture des postures des élèves : la diversité de leurs maniements cognitivo-langagiers.

\section{Bref rappel sur les postures d'étude des élèves}

Nous avons récemment complété l'inventaire des postures des élèves à l'occasion des multiples entretiens que nous avons eus avec des enseignants et assistants pédagogiques, lors de stage de formation (Bucheton Soulé 2009).

Nous avons ainsi identifié chez les élèves un jeu possible de 6 postures (réflexive, première, ludiquel créative, scolaire, de refus, dogmatique) traduisant les grandes caractéristiques de l'engagement des élèves dans les tâches.

Par posture première on décrit la manière dont les élèves se lancent dans la tâche sans trop réfléchir, laissant jaillir toutes sortes d'idées ou de solutions sans y revenir davantage. La posture scolaire caractérise davantage la manière dont l'élève essaie avant tout de rentrer dans les normes scolaires attendues, tente de se caler dans les attentes du maître. La posture ludique-créative traduit, elle, la tentation toujours latente et plus ou moins assurée de détourner la tâche ou de la re-prescrire à son gré.

La posture dogmatique manifeste une non-curiosité affirmée. Le "je sais déjà », le "mon ancien maître, ma mère, etc... me l'ont déjà dit ».

La posture réflexive est celle qui permet à l'élève non seulement d'être dans l'agir mais de revenir sur cet agir, de le «secondariser " pour en comprendre les finalités, les ratés, les apports. La posture de refus : refus de faire, d'apprendre, refus de se conformer est toujours un indicateur à prendre très au sérieux. Elle renvoie souvent à des problèmes identitaires, psychoaffectifs, à des violences symboliques ou réelles subies par les élèves.
Comme pour les postures des enseignants, les élèves circulent tous dans plusieurs postures d'étude. Ces postures ont toutes deux versants : un versant positif et un versant contre productif. Par exemple, sans posture première, il n'y a pas d'engagement rapide dans la tâche. Pour penser, il faut parfois d'abord essayer, brouillonner. À l'opposé, à trop secondariser, analyser, il n'y a parfois plus d'invention possible dans l'action. Savoir refuser est parfois une question de survie.

Aujourd'hui, les recherches menées à l'occasion du travail sur les ateliers dirigés conduisent à se demander si ce n'est pas le choix d'un jeu restreint de postures par les maîtres qui enfermerait les élèves dans un nombre lui aussi réduit de postures d'étude. Les premiers résultats obtenus apportent des éléments de confirmation à cette hypothèse.

\section{La posture d'étayage du maître : une organisation modulaire et finalisée de gestes}

Un premier constat s'est imposé très vite lors de nos analyses : si les élèves sont sensibles et réactifs à des gestes isolés (une réprimande, une demande d'explication, un sourire, le ton d'une réponse), ces micro-gestes ne modifient pas fondamentalement le cours de leur activité et la nature de leur engagement. En revanche, ils identifient très vite l'évolution ou les inflexions des attentes de l'enseignant pendant la leçon. Ils repèrent vite s'ils ont droit à l'erreur, si l'enseignant attend d'eux une réponse précise ou approximative, s'ils ont le droit de discuter avec leur camarade, de donner ou non leur point de vue, s'il faut écouter avec la plus grande attention ou simplement être en mesure de répéter, etc. Ils s'ajustent plus ou moins à ces attentes, parfois les refusent ou les détournent. Nous avons nommé «postures d'étayage » la diversité de ces conduites d'étayage de l'activité des élèves par les maîtres pendant la classe. Ce sont des organisations récurrentes de gestes faisant système, orientant et pilotant l'action des élèves de façon spécifique. Nous retrouvons donc notre définition première d'une organisation systémique et modulaire des gestes. Le choix des postures et de leur succession n'est pas aléatoire, ce que les auto-confrontations confirment. Les élèves savent rapidement décoder la grammaire implicite des postures possibles de leurs 
enseignants, la nature de leurs attentes, les changements d'étayage qu'elles manifestent.

\section{Un inventaire limité des postures d'étayage et leurs gestes constitutifs}

Les travaux menés pour l'instant sur la conduite des ateliers dirigés nous ont permis d'identifier plusieurs postures par lesquelles la nature de l'aide apportée par l'enseignant peut varier fortement.

- une posture de contrôle : elle vise à mettre en place un certain cadrage de la situation : par un pilotage serré de l'avancée des tâches, l'enseignant cherche à faire avancer tout le groupe en synchronie. Les gestes d'évaluation constants (feed-back) ramènent à l'enseignant placé en " tour de contrôle », la médiation de toutes les interactions des élèves. Les gestes de tissage sont rares. L'adresse est souvent collective, l'atmosphère relativement tendue,

- une posture de contre-étayage : variante de la posture de contrôle, le maître pour avancer plus vite, si la nécessité s'impose, peut aller jusqu'à faire à la place de l'élève.

- une posture d'accompagnement: le maître apporte, de manière latérale, une aide ponctuelle, en partie individuelle en partie collective, en fonction de l'avancée de la tâche et des obstacles à surmonter. Cette posture à l'opposé de la précédente ouvre le temps et le laisse travailler. L'enseignant évite de donner la réponse voire d'évaluer, il provoque des discussions entre les élèves, la recherche des références ou outils nécessaires. Il se retient d'intervenir, observe plus qu'il ne parle,

- une posture d'enseignement : l'enseignant formule, structure les savoirs, les normes, en fait éventuellement la démonstration. Il en est le garant. Il fait alors ce que l'élève ne peut pas encore faire tout seul. Ses apports sont ponctuels et surviennent à des moments spécifiques (souvent en fin d'atelier) mais aussi lorsque l'opportunité le demande. Dans ces moments spécifiques les savoirs, les techniques sont nommés. La place du métalangage est forte. Cette posture d'enseignement s'accompagne de gestes d'évaluation à caractère plutôt sommatif.

- une posture de lâcher-prise : l'enseignant assigne aux élèves la responsabilité de leur travail et l'autorisation à expérimenter les chemins qu'ils choisissent. Cette posture est ressentie par les élèves comme un gage de confiance. Les tâches données (fréquemment des fichiers) sont telles qu'ils peuvent aisément les résoudre seuls; les savoirs sont instrumentaux et ne sont pas verbalisés.

- une posture dite $d u$ " magicien » : par des jeux, des gestes théâtraux, des récits frappants, l'enseignant capte momentanément l'attention des élèves. Le savoir n'est ni nommé, ni construit, il est à deviner.

Dans le tableau suivant nous montrons comment chaque posture architecture de manière modulaire les divers gestes identifiés dans la matrice conceptuelle du multi-agenda. 
LES GeStes PROFESSiONNELS ET LE JEU DES POSTURES DE L'ENSEIGNANT DANS LA CLASSE...

Dominique Bucheton E Yves Soulé

\begin{tabular}{|c|c|c|c|c|c|}
\hline $\begin{array}{c}\text { Posture d'étayage } \\
\text { de l'enseignant }\end{array}$ & Pilotage & Atmosphère & Tissage & Objets de savoir & $\begin{array}{c}\text { Tâche élèves } \\
\text { postures }\end{array}$ \\
\hline Accompagnement & Souple et ouvert & $\begin{array}{l}\text { Détendue et } \\
\text { collaborative }\end{array}$ & $\begin{array}{l}\text { Très important } \\
\text { Multi directif }\end{array}$ & $\begin{array}{l}\text { Dévolution } \\
\text { Émergence }\end{array}$ & $\begin{array}{l}\text { "Faire et discuter } \\
\text { sur » : posture } \\
\text { réflexive, créative }\end{array}$ \\
\hline Contrôle & $\begin{array}{l}\text { Collectif } \\
\text { Synchronique } \\
\text { Très serré }\end{array}$ & $\begin{array}{c}\text { Tendue et } \\
\text { hiérarchique }\end{array}$ & Faible & En actes & $\begin{array}{c}\text { «Faire ": } \\
\text { Posture première }\end{array}$ \\
\hline Lâcher prise & $\begin{array}{c}\text { Confié au groupe, } \\
\text { autogéré }\end{array}$ & $\begin{array}{c}\text { Confiance, } \\
\text { refus } \\
\text { d'intervention du } \\
\text { maître } \\
\end{array}$ & $\begin{array}{c}\text { Laissé à l'initiative } \\
\text { de l'élève }\end{array}$ & En actes & $\begin{array}{l}\text { Variables : faire } \\
\text { Discuter sur }\end{array}$ \\
\hline $\begin{array}{c}\text { Enseignement } \\
\text { Conceptualisation }\end{array}$ & $\begin{array}{l}\text { Le choix du bon } \\
\text { moment }\end{array}$ & $\begin{array}{l}\text { Concentrée, } \\
\text { très attentive }\end{array}$ & $\begin{array}{c}\text { Liens entre les } \\
\text { tâches } \\
\text { Retour sur }\end{array}$ & Nommés & $\begin{array}{c}\text { Verbalisation } \\
\text { post-tâche } \\
\text { posture réflexive } \\
\text { (secondarisation) }\end{array}$ \\
\hline Magicien & $\begin{array}{l}\text { Théâtralisation, } \\
\text { mystère, révélation }\end{array}$ & $\begin{array}{l}\text { Devinette, } \\
\text { tâtonnement } \\
\text { aveugle, } \\
\text { manipulation }\end{array}$ & Aucun & Peu nommés & $\begin{array}{l}\text { Manipulations, } \\
\text { Jeu : posture } \\
\text { ludique }\end{array}$ \\
\hline
\end{tabular}

Figure 2 : Les postures d'étayage: une organisation modulaire de gestes et leurs visées

L'étude de nombreux ateliers d'écriture menés par des enseignants experts ou plus novices montre que jamais les enseignants ne se cantonnent dans une seule posture pendant le laps de temps d'environ une demi-heure que dure l'atelier. Pourtant certaines l'emportent sur d'autres. Ainsi, les jeunes stagiaires ont le plus grand mal à abandonner les postures de contrôle et d'enseignement même s'ils en ressentent les limites.

Nous observons aussi que la circulation entre ces diverses postures à l'intérieur de brefs scénarios est fréquemment l'apanage des maîtres très chevronnés sachant moduler les formes de leur «médiation " (en fonction de ce qu'ils perçoivent et observent de l'activité des élèves). Nous constatons souvent que ce souci de cadrage, de contrôle, de sur-étayage parfois est une préoccupation surdéterminante dans nombre de situations observées en ZEP. La posture dominante de cadrage et de contrôle développe alors une atmosphère très spécifique. Elle peut parfois volontairement être sur-jouée en début de cours chez les débutants pour permettre ensuite d'installer un accompagnement plus tranquille (RIA 2007).

\section{La dynamique réciproque des postures : un double ajustement}

L'ajustement des postures se fait à partir d'indicateurs perçus dans la dynamique de la situation : (bâillements de plus en plus fréquents, réponses inexactes, demandes d'aller aux toilettes, dépassement du temps prévu, agitation dans la classe ou au contraire silence pesant, etc.). Comme on change de braquet en vélo pour gravir une côte ou la redescendre, l'enseignant change de système d'étayage pour s'adapter à l'avancée de la leçon, aux difficultés perçues ou du moins à la représentation momentanée qu'il se fait de la situation. De la même façon, de leur côté, les élèves changent de posture en fonction de leur vécu, perçu de la difficulté pour eux de la tâche, en fonction aussi de la manière dont l'enseignant conduit le travail commun. Eux aussi se construisent pendant la leçon une représentation dynamique, plus ou moins proche de la réalité, de ce qu'ils peuvent ou veulent faire. Ces ajustements réciproques entre les changements de postures des enseignants et les postures d'apprentissage des élèves sont particulièrement visibles (effet loupe) dans le dispositif atelier à faible effectif. Il nous restait alors à nous demander s'il existait des invariants identifiables dans ces systèmes d'ajustements réciproques. Leur mise en évidence permettrait alors de comprendre certains mécanismes de « l'effet maître » ou de «l'effet élèves ». 


\section{Postures de l'enseignant}

\section{Postures des élèves}

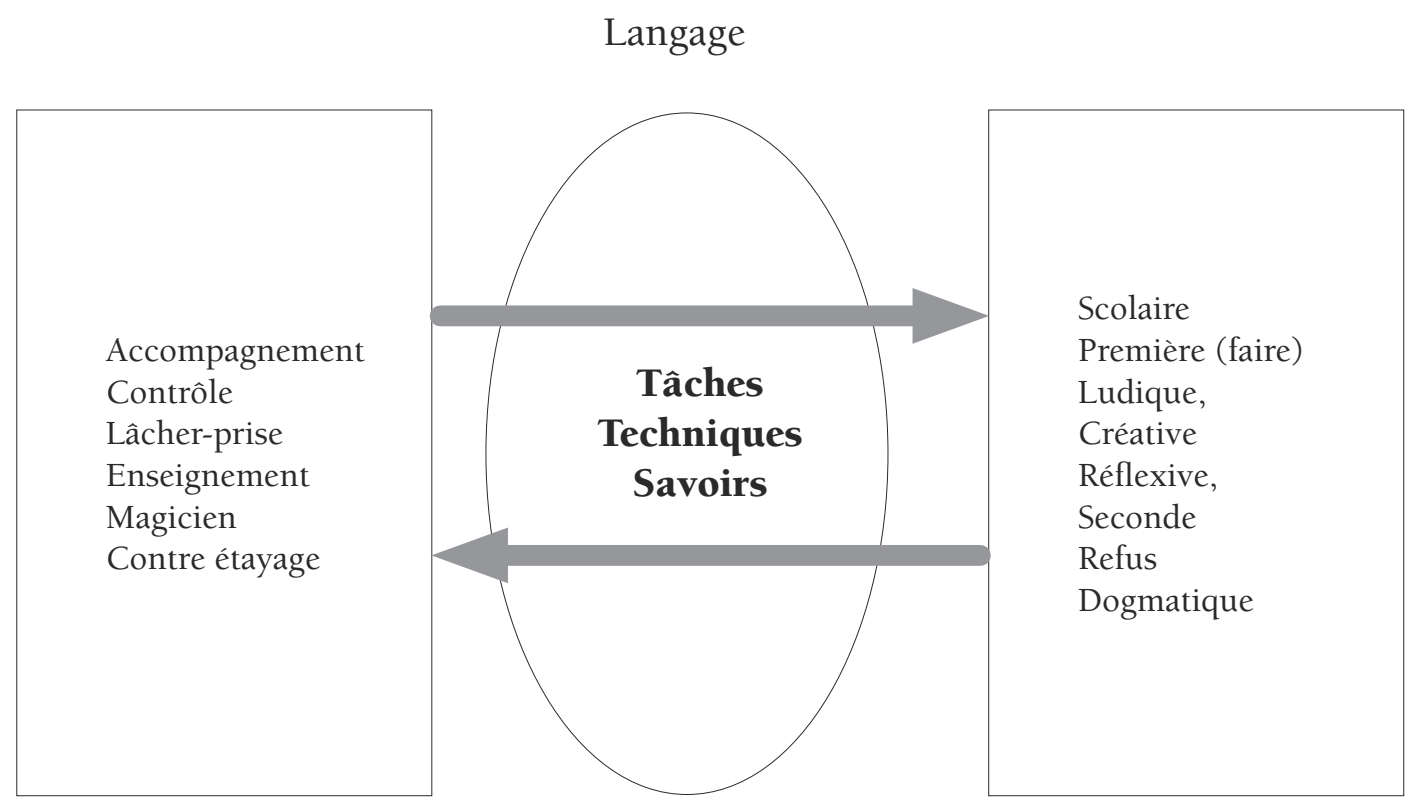

Figure 3 : Hypothèse de l'ajustement réciproque des postures des enseignants et des élèves

\section{Les changements de postures : le jeu entre dynamique de surface (contexte) et logiques profondes}

Les méthodologies multiples mises en œuvre par notre équipe ont permis d'avoir partiellement accès à ce que nous nommons les logiques d'arrièreplan constitutives de l'identité professionnelle et de l'éthique propre de chaque l'enseignant, marques aussi de sa singularité.

Les décisions de l'enseignant dans l'instant T sont ainsi le produit des contraintes de la dynamique de surface : les événements qui se sont produits dans le déroulement de la séance mais tout autant le fruit des cohérences, convictions ou dilemmes profonds qui se jouent à l'arrière-plan : un rapport conflictuel aux instructions de la discipline, une érudition dans un domaine de savoir une ignorance assumée ou non dans un autre, des souvenirs d'école douloureux, etc. Ces conflits ou cohérences profondes ne sont qu'en partie conscients; leur conceptualisation se fait fréquemment à l'occasion des entretiens ou des autoconfrontations croisées qui les font émerger après coup dans une post-réflexivité différente de celle qui est opérative pendant le cours de l'action.

L'ensemble de ces savoirs et logiques d'arrièreplan contribue à une représentation des possibles de la situation, lesquels permettent de convoquer les postures afférentes: des concepts pragmatiques, une réflexivité en actes qui entre dans le jeu de l'agir partagé. De sorte qu'enseigner est toujours un compromis entre sa propre culture, les savoirs que l'expérience a accumulés, les gestes, artefacts et petites méthodes que l'on a mis au point et le devenir toujours incertain du déroulé de la séance : «Aujourd'hui rien à faire, ça dort! Ils ne sont pas là! ", dit une collègue très experte lors d'un entretien.

Postures d'étayage des enseignants, postures d'étude des élèves : quels liens? Une étude de cas

Nous n'avons pas encore mis en évidence de manière évaluée les liens entre les postures des enseignants et celles des élèves. Les travaux menés actuel- 
lement en collège ZEP montrent des corrélations fortes : un jeu de postures plus ouvert, le maintien plus long en posture réflexive chez les élèves lorsque les enseignants sont davantage dans la posture d'accompagnement lors des ateliers. Mais il reste à évaluer les effets de ces attitudes sur les résultats et le développement des élèves (projet de recherche en cours). Nous nous contenterons donc de présenter un cas ponctuel : la comparaison de l'agir de deux enseignantes lors d'un atelier d'écriture en CP et ce qu'il nous permet de comprendre sur les mécanismes de la co-activité enseignant - élèves. L'exemple est heuristique. Il sera ici brièvement présenté pour montrer comment la modélisation proposée permet de comparer deux scénarios en apparence identique chez deux enseignantes et permet d'en analyser les effets induits sur les élèves.

Il s'agit pour les deux enseignantes de décider du texte qui va être écrit collectivement pendant la durée de l'atelier (trente minutes). Isabelle conduit de tels ateliers depuis longtemps, Sophie, bien qu'enseignante très expérimentée est novice pour ce dispositif spécifique. C'est la deuxième fois qu'elle le pratique.

Isabelle : une posture d'accompagnement dominante dans le premier scénario

Le scénario dure 7 minutes chez Isabelle et un peu moins chez Sophie. Isabelle laisse les élèves lentement et tranquillement inventer le texte en leur donnant toute liberté pour tisser toutes sortes de liens avec leur famille, avec ce qu'ils ont déjà écrit, avec la nature du projet d'écriture. L'atmosphère est détendue, miludique, mi-sérieuse. L'enseignante plaisante, sourit. Assise parmi les élèves, elle est occupée à tranquillement défaire le film plastique qui couvre une gomme. Elle est dans une posture d'accompagnement : elle veille cependant à garder la direction du récit inventé, ce n'est pas de bouchons qu'on a besoin mais de pots de yaourts, fait répéter la phrase plusieurs fois, explique ce que les élèves ne peuvent pas savoir : «Eh bien moi je vais vous le dire, vous ne pouvez pas le savoir, on va faire un premier jet, on va chercher les mots et puis on écrira joli et on signera tous (geste imaginaire sur la feuille). Les élèves sont avec elle, ils se sont engagés dans ce qui va être leur histoire, leurs problèmes à résoudre. Et quels problèmes! En 35 minutes, ils vont écrire (sauf le dernier mot), eux dont certains ne connaissent pas même encore toutes les lettres: Bonjour papa et maman, est-ce que vous pouvez nous donner des pots de (yaourts).

Dans ce premier scénario et dans les suivants la posture d'accompagnement domine. Elle cédera la place en fin de séance à une posture d'enseignement et d'évaluation : moment où l'enseignante fait le bilan avec chaque élève de ce qu'il a réussi, essayé ou raté. « Des pots : là il faut un $S$, il en a plusieurs, et toi Zélie, il faut qu'on revoie le $P$ et le B en remédiation.

Dans l'entretien Isabelle explique :

Enquêteur: au départ de l'atelier, tu savais que tu voulais leur faire écrire à tous la même phrase?

- Isabelle : Non/ non/ je ne sais jamais/mais ça peut varier. Faut pas que ce soit ma phrase à moi/ parce que c'est toujours le danger/ ils sont en début de CP. L'idée c'est qu'ils aient envie d'écrire et puis qu'ils aient du plaisir à le faire/ mais tout ce qui se passe au niveau de la langue orale c'est essentiel aussi hein/ ils vont/ ils négocient entre eux (...)

En même temps je les mets en situation del de futurs écrivains : je sais pas (rires...) alors là l'idée hein / c'est le brouillon hein, / c'est que dans la vie on fait des brouillons et puis euh// parce que je sais pas/ pour avoir écrit en groupe d'adultes je sais que c'est hyper difficile de négocier parfois un mot /

(...) Je suis persuadée que / l'écriture/ la lecture c'est lié à l'affectif et le nier ça serait/ ça serait oui ça serait même pas pédagogique je trouve.

Sa posture d'accompagnement est aussi sous-tendue (niveau des logiques d'arrière-plan) par une théorie de l'apprentissage : l'idée qu'il faut amener les élèves à s'essayer, à chercher à résoudre un problème au-delà de ce qu'ils savent déjà, en sachant que son rôle est alors de les accompagner. Les laisser partir à l'aventure mais en restant extrêmement vigilante et présente. Isabelle est très consciente de cet équilibre fragile!

Et là je me dis tout le temps parle pas trop, parle pas trop.....

Voilà oui/ c'est/ en fait c'est toujours en tension entre ma place, pas ma placel est-ce que c'est trop/ je 
me réajuste sans cesse quoi/ j'essaie de/ d'aller à leur rythme pour que ce soit une expériencel aussi un bon souvenir pour eux.

Les postures des élèves d'Isabelle : pendant tout ce premier scénario et dans ceux qui suivent d'ailleurs les 5 élèves oscillent entre des postures ludique-créative et réflexive. Les gestes d'étude mis en place sont aussi bien cognitifs (inventer, planifier un texte, choisir une énonciation, se souvenir de que l'on sait déjà écrire) que sociaux (s'autoriser à parler de soi, écouter les autres, négocier avec eux le texte à écrire).

Nous identifions lors de ce premier scénario une relation entre la posture d'accompagnement du maître et les postures réflexive et créative des élèves. Nous n'observons aucun élève en posture de refus. L'hétérogénéité du groupe est positive et dynamique.

Sophie : une posture de contrôle dominante, la parole des élèves en partie confisquée

Dans ce premier scénario, beaucoup plus court ici (3 minutes), Sophie de la même façon doit négocier le texte à écrire. Elle se heurte à une difficulté. Elle veut faire écrire la suite d'un texte écrit par un autre groupe (projet d'article de journal scolaire sur la visite au Corum de Montpellier) mais les élèves eux ont envie de raconter ce qu'ils ont vécu lors de leur visite. Elle tranche et impose sa propre phrase. «Nous avons pris le bus»

L'ensemble des gestes de Sophie nous amène à penser qu'elle est le plus souvent dans une posture de contrôle des élèves, de contrôle de l'avancée de la tâche, des contenus qui y seront travaillés. Dans ce premier scénario d'invention planification du texte, son souci premier semble être le pilotage visant à arriver très vite vers l'épisode suivant: le corps à corps, lettre après lettre avec l'écriture. Du coup, la parole de certains élèves est confisquée, l'atmosphère est contrainte, les opérations premières de l'écriture (organiser une pensée, la mettre en mots) sont écrasées au profit d'une centration qui va suivre sur l'encodage des sons. C'est l'enseignante au final qui impose le texte à écrire. La suite de la séance la verra occuper une posture fréquente d'enseignement appuyée sur des gestes d'évaluation.
Ses représentations des possibles de l'atelier d'écriture, des possibles de ses élèves, sont différentes. À ce moment-ci de l'année et de sa formation en cours (sur les ateliers d'écriture) elle cadre, pilote, régule pas à pas l'activité des élèves dans une forme de sur-étayage dont elle est consciente. Elle la justifie par son expérience : «En ZEP, il faut cadrer beaucoup ». Cette représentation est fréquente. Elle justifie aussi cette mise sous tutelle de la parole et de la pensée des élèves par son souci de pilotage (faire avancer la séance) et l'accrocher au travail du groupe précédent. "Moi mon projet, en fait, j'avais déjà un projet dans la tête, c'était que la deuxième phrase devait concerner les transports. Donc j'ai essayé de recadrer pour que la phrase soit sur ce sujet-là; (...) J'oriente ça c'est clair... Que les élèves s'expriment ça c'est très bien mais on ne va pas en une séance pouvoir tout dire ce qu'ils ont envie de dire (...) Il faut que je recadre : dans un premier temps, on part du transport, ensuite il faut décrire ce qu'on a vu, ensuite ce qu'on a entendu, donc euh, la structure du texte c'est moi qui l'ai en tête, c'est clair ». Le dilemme didactique classique (centration sur le résultat: la production d'un texte achevé plus que sur le processus) peut aussi en partie expliquer les stratégies adoptées.

Cette posture de contrôle est aussi ici justifiée par un souci de différenciation. Commentant ses déplacements pour correction, derrière les élèves, elle dit : "J'essaie d'individualiser l'aide que j'apporte à chacun: pour l'un, lui apprendre à espacer les mots, pour l'autre montrer la graphie d'une lettre ».

Elle s'accompagne cependant pour deux élèves d'une posture que nous avons qualifiée de « lâcher prise »: "Quand on a un groupe comme celui-ci, qui est un groupe trop hétérogène: la différence entre Joachim et Hanam qui trouvent très vite comment écrire et les autres qui rament un peu, cette différence est trop importante. J et $H$ étaient tellement à l'aise que ça empêche les autres de chercher. Je me suis dit: tous les deux sont lancés, je les envoie en autonomie ».

Les postures des élèves de Sophie :

Dans ce cours scénario, on observe diverses postures chez les élèves : la créativité et l'engagement qu'ils manifestent d'abord dans le désir de raconter s'éteignent très vite. Des formes de refus se manifestent par un léger chahut de deux élèves ou par le 
silence des autres. Tous rentrent momentanément en posture scolaire : les normes dont données. Il faut écrire « en attaché » dans « le rail » dessiné au préalable par l'enseignante sur la feuille.

La configuration dominante de ce premier scénario montre une corrélation entre une posture de cadrage /contrôle forte et des postures faiblement réflexives des élèves. Ce cadrage fort de l'atelier à son début installe ici la posture scolaire, voire momentanément une posture de refus. Deux élèves en avance s'en échappent. L'hétérogénéité du groupe n'est plus une ressource mais devient un obstacle que l'enseignante n'arrive à gérer qu'en excluant (positivement) les deux élèves.

\section{Conclusions et perspectives :}

Une grammaire de l'agir enseignant à poursuivre D'une description statique des composantes de l'agir enseignant (l'ensemble de cinq préoccupations organisatrices de son action), nous avons proposé une conceptualisation de l'organisation modulaire, systémique et dynamique des gestes professionnels (le jeu des postures d'étayage des maîtres). Celle-ci éclaire en partie la dynamique du sens et de l'engagement des acteurs dans le déroulement de la situation. Elle contribue à repenser l'effet maître. Les entretiens et auto-confrontations montrent aussi que cette dynamique de « surface » est sous-tendue par un système de représentations et conceptualisations d'arrièreplan assez rarement questionnées en formation et pourtant décisives. L'ensemble du réseau de concepts que ces deux modélisations proposent ouvre des perspectives importantes sur l'identification des liens entre les GP et postures d'étayage du maître et les gestes d'étude et postures des élèves dans des situations didactiques précises.

Les deux niveaux de conceptualisation proposés devraient permettre de mieux identifier les conditions favorables du développement des élèves. En ce sens elles s'avèrent des instruments pour la formation.
Mais une grammaire à poursuivre. L'étude de l'agir enseignant ne peut être véritablement convaincante que si elle ausculte attentivement comment ces diverses préoccupations, postures d'étayage s'actualisent dans les diverses situations didactiques, comment elles mettent en travail diverses postures ou gestes d'étude spécifiques des disciplines, comment elles permettent de résoudre des problèmes d'apprentissage des élèves. C'est en travaillant au plus près des préoccupations didactiques du cycle 2 en français que nous avons aperçu la densité de cet agir, qui articule toujours les dimensions sociales, interactionnelles, langagières, éducatives, didactiques et cognitives.

D'où une triple visée pour la recherche et la formation

-un observatoire pour approcher mieux la complexité et la singularité de l'agir enseignant ordinaire, sa dynamique, ses ajustements constants en situation et dans l'action.

-un conservatoire: pour identifier, nommer, classer les grandes formes, genres, modèles didactiques construits dans la longue histoire de la culture professionnelle. En repérer les points communs et variations. Permettre la compréhension fine des raisons pour lesquelles, selon les contextes ils fonctionnent ou dysfonctionnent.

-un chantier pour l'invention technologiquement raisonnée, d'une nouvelle professionnalité, de nouvelles formes scolaires, de nouvelles situations, de nouvelles tâches qui répondent aux contextes nouveaux, publics nouveaux, savoirs nouveaux auxquels le métier enseignant va devoir s'adapter. Les travaux exploratoires que nous menons actuellement avec des enseignants au collège et en cycle 2 sur l'atelier dirigé comme possible stratégie pour répondre à la très grande hétérogénéité des élèves ${ }^{6}$ vont dans cette visée d'élaboration de pratiques alternatives, accompagnées technologiquement par la recherche. 


\section{NOTES}

1. Équipe dirigée par Dominique Bucheton et composée de : Accardi Jocelyne, Asdhi Carole, Bronner Alain, Brénas Yolande, Broussal Dominique, Brunet Lisa Marie, Chabanne J. Charles, Decron Alain, Deyrich M.Christine, Dupuy Catherine, Etienne Richard, Jean Alain, Jorro Anne, Jaubert Martine, Larguier Myrène, Liria Aurélie, Pelissier Chrysta, Rebière Maryse, Soulé Yves, Veyrunes Philippe

2. Nous (Bucheton, Jorro, p. 7-24) avons longuement développé nos sources théoriques et leur articulation dans le premier chapitre de l'ouvrage collectif : L'agir enseignant : une question d'ajustement, Bucheton (dir), Octares (2009)

3. Journées d'étude de Lyon : la construction de la référence 2006, organisées par l'association internationale de recherche en didactique comparée (ARCD).

4. Cette question de la gestion des imprévus par les enseignants débutants est apparue si importante pour l'équipe qu'elle a fait l'objet de la thèse d'Alain Jean 2008

5. L’analyse du développement professionnel auquel a donné lieu ce stage a été réalisée dans le cadre d'un mémoire du Master Professionnel Conseil et formation, Montpellier III : Aurélie Liria (2005) Premiers pas dans la lecture écriture au CP. Professionnaliser des formés et professionnalisme des formateurs

6. Ces travaux, menés avec la collaboration étroite d'enseignants, sont conduits en partenariat avec l'Institut National de la Recherche Pédagogique. Ils s'inscrivent dans le programme de recherche de l'INRP dirigé par Luc Ria. «Vers une nouvelle professionnalité des enseignants». 


\section{BIBLIOGRAPHIE}

Bakhtine, M. (1984). Esthétique de la création verbale. Paris : Gallimard.

Bautier, E. (1995.) Pratiques langagières, pratiques sociales. Paris : l'Harmattan.

Berthoz, A. (2003) La décision, Paris, Odile Jacob

Blanchard-Laville, Cl. (dir) (2003). Une pratique ordinaire, Mélanie, passe au tableau, Paris : L'Harmattan.

Broussal, D. (2006). Interagir en début de cours : une professionnalisation du malentendu, entre savoir et langage, Thèse de doctorat, Université Paul Valéry, Montpellier, non publiée.

Bruner, J. (1984). Contextes et formats. In M. Deleau (dir.), Langage et communication à l'âge préscolaire. Presses universitaires de Rennes 2, (p. 13-26).

Bruner, J. (2003) Le développement de l'enfant : savoir-faire, savoir-dire, Paris : PUF,

Bruner, J. (2000) Culture et modes de pensée. L'esprit humain dans ses œuvres, Paris : Retz

Brunet, L.-M., Liria, A. (2004). Variation sur une même partition : étude des gestes professionnels de deux enseignants de CP travaillant avec la même méthode de lecture, Ratus et ses amis. Mémoire de maîtrise de Sciences de l'éducation (non publié) sous la direction de D. Bucheton, Université Paul Valéry 3, Montpellier.

Bucheton, D., (1994), « Vécu, affects et didactique du récit », Le Français Aujourd'hui, Nº 109.

Bucheton D., Bautier E., (1997), Conduites d'écriture au collège et LP Paris : Delagrave

Bucheton, D,. (1999), « Les postures du lecteur », pp. 137-150 in Lecture privée, lecture scolaire, la question de la littérature à l'école, Demougins P,. Massol, JF. (ed) CRDP Grenoble.

Bucheton, D., Bronner, A., Broussal, D., Jorro, A. § Largier, M. (2004) Les pratiques langagières des enseignants : des savoirs professionnels inédits en formation, Repères, 30, 33-53, Paris, INRP.

Bucheton, D., Brunet, L.-M., Liria, A. (2005). L'activité enseignante, une architecture complexe de gestes professionnels. Dans CD-Rom Former des enseignants professionnels, savoirs et compétences, Colloque Nantes, M. Altet (ed)

Bucheton, D., (2006). Les postures d'écriture et de lecture : la diversité des modes de penser-parler-apprendre, Lausanne : Langage \& Pratiques, 37, 29-39.

Bucheton, D., (2007) Didactique Professionnelle, didactique disciplinaire, le rôle intégrateur du langage, Didactique disciplinaire, didactique professionnelle; in Lenoir, Y., Pastré, P. (eds), Toulouse : Octares,

Bucheton, D., (dir) (2009 A). L'agir enseignant: des gestes professionnels ajustés, Toulouse : Octares.

Bucheton, D., Soulé, Y. (2009 B) L'atelier dirigé d'écriture au CP : une réponse à l'hétérogénéité des élèves. Delagrave : Paris

Chabanne, J.-C., Bucheton, D. (dir.), (2002). Parler et écrire pour penser, apprendre et se construire. Paris : PUF

Chevallard, Y. (1999). L'analyse des pratiques enseignantes en théorie anthropologique du didactique, RDM 19 février. Grenoble, La Pensée Sauvage.

Clot, Y., (1999). « Le geste est-il transmissible?» Apprendre autrement aujourd'hui, Dixièmes entretiens de la Villette. 24 au 26 novembre 1999.

Decron, A. (2007). Le récit éducatif un tissage discursif complexe pour apprendre, penser et se construire. Thèse de doctorat, Université de Montpellier III.

Crinon, J., Marin, B., Bautier, E. (2008) Quelles situations de travail pour quel apprentissage? Paroles des élèves, paroles de l'enseignant in Le développement des gestes professionnels dans l'enseignement du français, un défi pour la recherche et la formation, Bucheton, D., Dezutter, O. (Dir)

Damasio, A (2001). Le Sentiment même de soi. Corps, émotions, conscience. Paris, Odile Jacob.

Broussal, D. (2006) Interagir en début de cours : une professionnalisation du malentendu, entre savoir et langage, Thèse non publiée, sous la direction de D. Bucheton, Montpellier 3. 


\section{LES GESTES PROFESSIONNELS ET LE JEU DES POSTURES DE L'ENSEIGNANT DANS LA CLASSE...}

\section{Dominique Bucheton \& Yves Soulé}

Durand, M,. (1996). L'enseignement en milieu scolaire, Paris, PUF.

Clot, Y. (1999) La fonction psychologique du travail, Paris, PUF

François, F., (dir.), (1990). La communication inégale. Heurs et malheurs de l'interaction verbale. Paris, Delachaux et Niestlé.

Goffman, E. (1901). Les cadres de l'expérience, Paris, Minuit.

Jaubert, M., Rebière, M. (2001), « Pratiques de reformulation et construction des savoirs ", Aster, № 33, 81-110.

Jaubert, M. (2001) « Cohérence textuelle et positionnement énonciatif contextuellement pertinent en classe de sciences » in J.P. Bernié, (dir), Apprentissage, développement et significations, Presses universitaires de Bordeaux, 173-190.

Jean, A. (2008) : Le traitement des imprévus par des professeurs de technologie en formation initiale à l'IUFM. Quels gestes d'ajustement en situation de classe? Quelle utilisation pour le développement professionnel? Thèse de doctorat non publiée, Université Montpellier 3, sous la direction de R. Etienne.

Jorro, A., (2002), Professionnaliser le métier d'enseignant. Paris, ESF.

Jorro, A., (2004), « Le corps parlant de l'enseignant. Entente, malentendus, négociations », Symposium : La réflexivité des langages, instruments de travail du professeur et des élèves : points communs et spécificité disciplinaires, AIRDF, Québec (CD-ROM)

Labov, W. (1976). Le parler ordinaire, Paris : Minuit

Mac Court, Frank (2006). Teacher Man, Harper Perennial, London.

Pennac, D. (2007). Chagrins d'école. Pris : Gallimard.

Matheron, Y. (2000). Une étude didactique de la mémoire dans l'enseignement des mathématiques au collège et au lycée, quelques exemples, Université Aix-marseille I, Université de Provence.

Perret-Clermont, A.M. (1979). La construction de l'intelligence dans l'interaction sociale, Genève : P. Lang

Pastré, P. (2002), L’analyse du travail en didactique professionnelle, Revue Française de pédagogie. № 138,

Pastré, P., Mayen, P. ,Vergnaud, G. (2006). la didactique professionnelle, Revue Française de Pédagogie, 154, $145-198$

Saada-Robert, M. § Baslev, K. (2004), « Une micro-génèse située des significations et des savoirs », in Moro, C., Rickenman, R. (eds), Situation éducative et significations, 135-164, Bruxelles : De Boeck.

Soulé, Y., Bucheton, D. (2007). Les Gestes professionnels d'évaluation, postures d'étayage et leurs effets dans la classe de français. Communication présentée symposium REF : Les gestes d'évaluation dans la classe de français, Sherbrooke, Québec : à paraître De Boeck.

Sensévy, G., Mercier, A. (2007). Agir ensemble : l'action didactique conjointe du professeur et des élèves, Rennes : Presses universitaires de Rennes.

Sensévy, G., (2008), «Le travail du professeur pour la théorie de l'action conjointe en didactique. Une action située », pp. 39-51, in Recherche et formation : Le travail enseignant: Crises et recomposition, du local à l'international, retour sur le métier, Nº 57 Lyon, INRP.

Thévenaz-Christen, Th. (2005). Les prémices de la forme scolaire. Études d'activités langagières orales à l'école enfantine genevoise. Thèse de doctorat de l'université de Genève.

Ria, L, Chalies, S. (2003). Dynamique émotionnelle et activité : le cas des enseignants débutants, INRP, Recherche et Formation, 42, p 7-19

Ria, L. (2007). Devenir enseignant : parcours et formation, Paris : A Colin.

Vergnaud, G. (1996), «Au fond de l'action, la conceptualisation », in J.-M. Barbier (ed) Savoirs théoriques, savoirs d'action. Paris : PUF.

Vygotski, L.(1985). Pensée et langage. Paris, Messidor, éd. sociales.

Vincent, J.D., (2007), Voyage extraordinaire au centre du cerveau, Paris : O. Jacob. 